\title{
New insights into the proximal tendons of adductor longus, adductor brevis and gracilis
}

\author{
J A Davis, M D Stringer, S J Woodley
}

Department of Anatomy, Otago School of Medical Sciences, University of Otago, Dunedin, New Zealand

\section{Correspondence to}

Stephanie J Woodley,

Department of Anatomy,

Otago School of Medical

Sciences, University of Otago,

P0 Box 913, Dunedin 9054,

New Zealand:

stephanie.woodley@anatomy. otago.ac.nz

Received 17 March 2011 Accepted 8 September 2011

Published Online First 17 October 2011

\begin{abstract}
Objective The adductor muscles are implicated in the pathogenesis of groin strain, but the proximal morphology of this muscle group is poorly defined. The purpose of this study was to investigate the detailed anatomy of the entheses and proximal musculotendinous junctions (MTJs) of adductor longus (AL), adductor brevis (AB) and gracilis.
\end{abstract}

Methods The adductors were dissected bilaterally in 10 embalmed cadavers $(7$ females, mean age at death 79 years (range 57-93 years)), focusing on the type of enthesis, and architecture of the proximal tendons and MTJs. Histology was used to determine if the entheses contained fibrocartilage and to semiquantitatively assess the vascularity of each proximal tendon.

Results All entheses were fibrocartilaginous. The proximal tendons of $A B$ and gracilis were fused, forming a common tendinous insertion in all specimens. AL and $A B$ both contained extensive intramuscular tendons of variable length $(A L 11.1 \pm 1.5 \mathrm{~cm} ; A B 5.4 \pm 1.1 \mathrm{~cm})$; this has not been recorded previously. The vascularity of $A L$ and $A B$ tendons decreased significantly towards the enthesis $(p<0.05)$, and their entheses were significantly less vascular than that of gracilis $(p<0.05)$.

Conclusions The proximal anatomy of $A L, A B$ and gracilis is more complex than previously described. The arrangement and fusion of these muscles, their fibrocartilaginous entheses and differences in vascularity of their proximal tendons may be important anatomical considerations in the pathogenesis and pattern of adductorrelated groin pain.

\section{INTRODUCTION}

Groin pain is a common complaint in athletes, and particularly prevalent in sports that involve repetitive twisting, kicking and rapid changes in direction, such as football, ice hockey and Australian football.1-6 Studies on professional football players quote a prevalence of up to $12 \%,{ }^{37}$ and an incidence of two adductor injuries per 1000 player-hours. ${ }^{4}$ Differential diagnosis of this complex, multifactorial disorder ${ }^{8}$ is often challenging due to the lack of consensus regarding both nomenclature and diagnostic criteria. ${ }^{9}$ Despite this, it is generally accepted that groin pain can be classified as either acute or chronic. ${ }^{10}$ Acute injuries encompass a variety of potential pathologies such as adductor tendinitis, muscle tears and strains, and inguinal hernia. ${ }^{811} 12$ Acute rupture or avulsion of a proximal adductor tendon is rare ${ }^{1314}$ Chronic groin pain can result from conditions such as osteitis pubis, tendinopathy, enthesopathy, hip joint pathology and 'sportsman's hernia'.11 12 15-20
Diagnosis of adductor-related groin pain is usually based on clinical findings. ${ }^{19} 21$ Consequently, few studies have reported specifically what muscles are involved and their precise site of pathology. Although the adductors are often referred to as a group, several sources state that, from clinical examination, adductor longus (AL) is most often implicated in adductor-related groin pain. ${ }^{18-2022-25}$ In some cases, AL pathology has been confirmed by MRI, which has shown pathology at the enthesis alone, ${ }^{2025}$ or distributed across the enthesis, proximal tendon and muscle. ${ }^{26}$ Involvement of adductor brevis $(\mathrm{AB})$ and gracilis in this condition has been reported less often. ${ }^{21726-28}$ Surprisingly, no study appears to have systematically investigated all these muscles in affected individuals. Similarly, little attention has been given to specifying the precise anatomical location of adductorrelated groin pain: the proximal enthesis, 15182025 proximal tendon, 11121516 musculotendinous junction $(M T J)^{16} 1827$ and muscle ${ }^{11}$ have each been identified as actual or potential sites of injury. However, with the exception of one study ${ }^{15}$ no data exist on the relative frequencies of injury at these various sites.

Accurate morphological description of the proximal adductor muscles is essential if we are to enhance our understanding of adductor-related groin pain in general and to improve clinical diagnosis, interpretation of imaging investigations, and injury prevention and treatment strategies in particular. Given the most commonly reported site of injury, surprisingly few studies have investigated the proximal anatomy of the adductors; ${ }^{14} 29-31$ only one study has considered this muscle group in its entirety. ${ }^{30}$ The MTJ of AL has been examined in just two studies, which reported on its outer appearance ${ }^{32}$ and crosssectional area (CSA) of the proximal tendon. ${ }^{31}$ Detailed descriptions of the MTJ and proximal tendon of $A B$ and gracilis are lacking. Proximal fusion of muscles could affect patterns of injury but the connection between AL and gracilis is controversial; some reports state that the tendons are fused, ${ }^{2} 3334$ whereas anatomy reference texts typically maintain that they are separate. ${ }^{35-37}$ With regard to $A B$, it has been reported that only a small portion of its tendon is visible on the external surface of the muscle, ${ }^{30}$ which suggests the presence of an intramuscular tendon but this has yet to be confirmed. Since some cases of adductor-related groin pain may arise from an enthesopathy or tendinopathy, ${ }^{11}{ }^{15-17} 1920$ knowledge of enthesis composition and tendon vascularity also seem relevant. With the exception 
of a small case series indicating that the enthesis of AL is fibrocartilaginous, ${ }^{14}$ no other information is available on this subject. The vascularity of these tendons could influence their susceptibility to injury and ability to repair. It is well known that tendons heal slowly after trauma ${ }^{38}$ and relatively poor vascularity could contribute to adductor pathology. ${ }^{21}$ While it has been hypothesised that the anterior region of the proximal tendon and MTJ of AL is poorly vascularised, 31333439 the vascularity of the adductor tendons and their MTJs has not been formally investigated.

The aims of this exploratory study were to examine the proximal anatomy of $\mathrm{AL}, \mathrm{AB}$ and gracilis specifically to determine the architecture of their tendons and MTJs, ascertain the extent of fusion between these muscles, establish the composition of their entheses and investigate the relative vascularity of their tendons and MTJs.
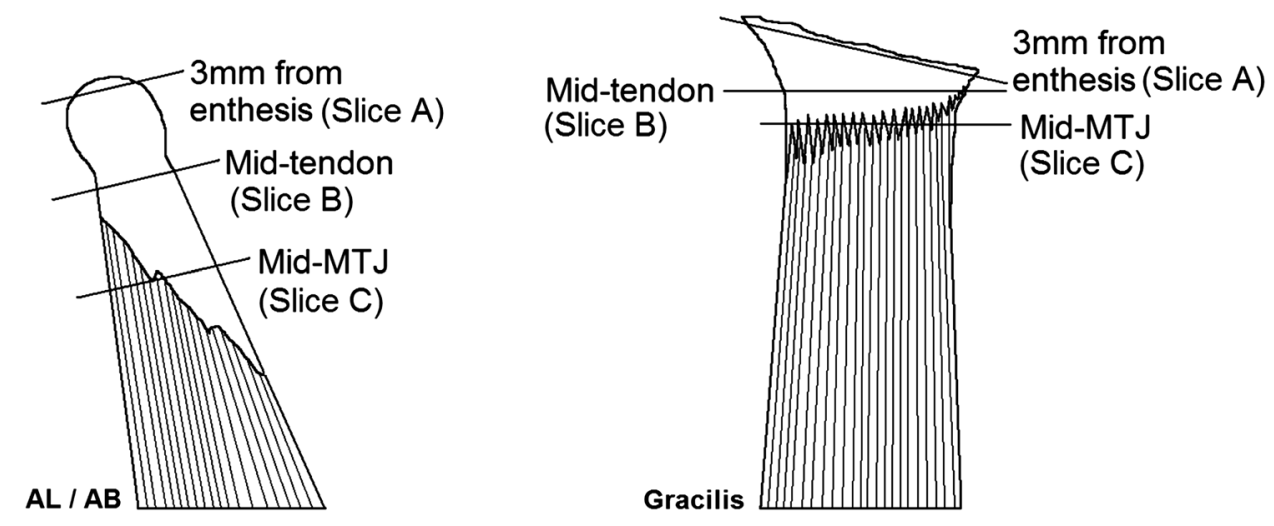

Figure 1 Schematic showing sites of transverse sections taken through the proximal tendons of $A L, A B$ and gracilis. Slice $A$ at $3 \mathrm{~mm}$ from enthesis, slice $B$ at mid-tendon, and slice $C$ at mid-MTJ. AB, Adductor brevis; AL, adductor longus; MTJ, musculotendinous junction.
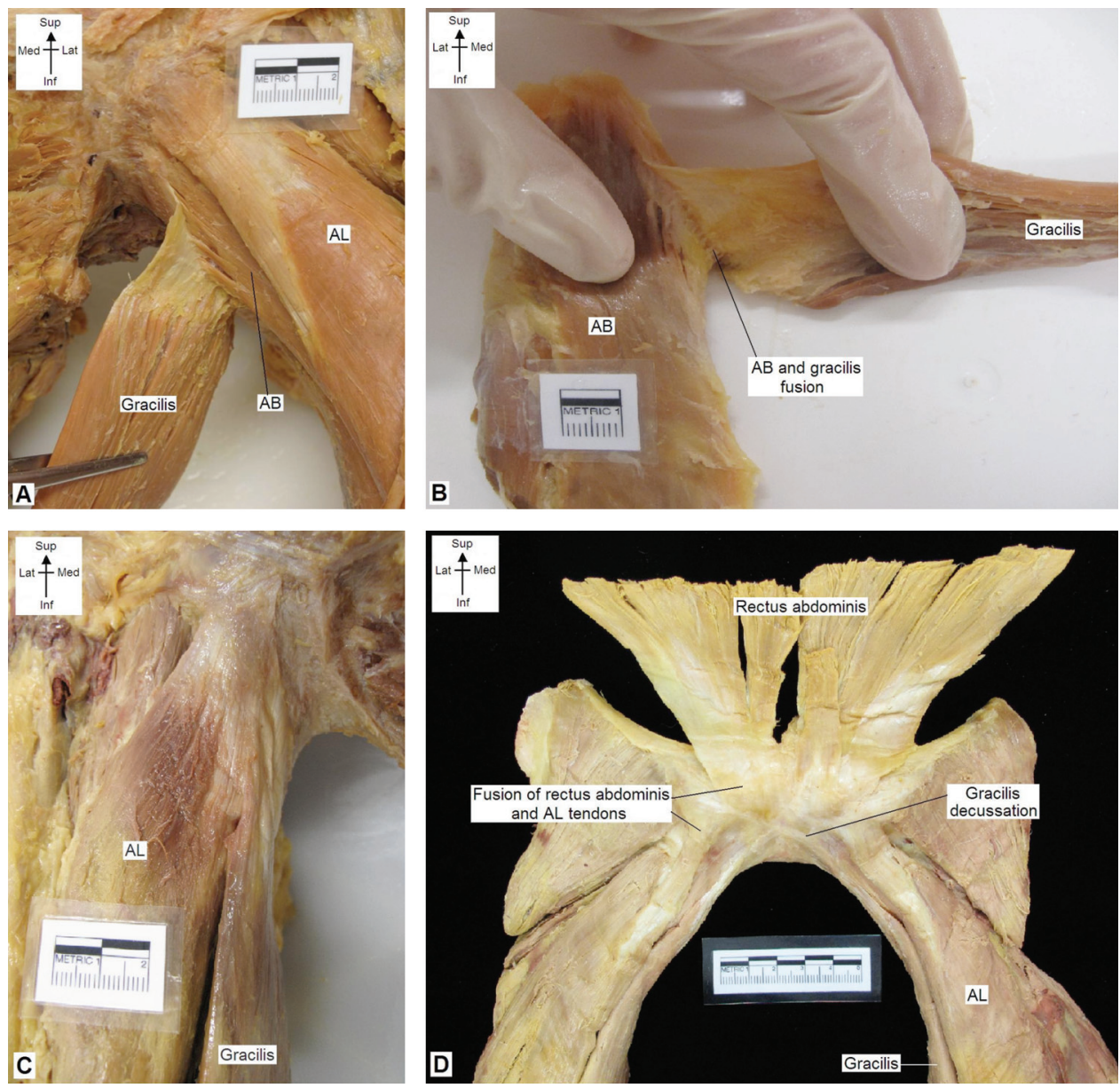

Figure $2(A-D)$ Representative images of proximal adductor fusion. (A) $A B$ and gracilis fusion showing $A B$ muscle fibres inserting into the deep aspect of the gracilis tendon (male specimens, $n=6 / 6$ ). (B) Proximal fusion between the tendons of $A B$ and gracilis (female specimens,

$n=14 / 14)$. (C) Fusion of the proximal tendons of $A L$ and gracilis $(n=2 / 20)$. (D) Decussation of the proximal tendons of gracilis $(n=12 / 20)$ and fusion between the tendons of $A L$ and rectus abdominis $(n=20 / 20)$. $A B$, adductor brevis; $A L$, adductor longus. 
Table 1 Anatomical parameters of adductor longus, adductor brevis and gracilis

\begin{tabular}{lcll}
\hline & Adductor longus & Adductor brevis & Gracilis \\
\hline Footprint & & & \\
$\quad$ Shape & Oval & Oval & Rectangular \\
$\quad$ Dimensions* & $1.5 \pm 0.2 \times 1.9 \pm 0.3$ & $1.3 \pm 0.2 \times 2.4 \pm 0.5$ & $5.4 \pm 1.0 \times 0.4 \pm 0.1$ \\
Tendon length & & & \\
$\quad$ Surface & $7.7 \pm 1.1$ & $3.6 \pm 0.6$ & $5.5 \pm 1.0$ \\
$\quad$ Intramuscular & $11.1 \pm 1.5$ & $5.4 \pm 1.1$ & Not present \\
MTJ length & $9.7 \pm 1.1$ & $4.1 \pm 0.6$ & $4.8 \pm 0.7$ \\
\hline
\end{tabular}

All measurements in $\mathrm{cm}$.

*Footprint dimensions $=$ maximum height $\times$ maximum width.

MTJ, musculotendinous junction.

\section{MATERIALS AND METHODS}

Using a combination of dissection and histology, the adductor muscles were examined by one investigator (JAD) in 10 embalmed cadavers (20 lower limbs), bequeathed to the University of Otago under the New Zealand Human Tissue Acts (1964 and 2008). Seven cadavers were female and three were male, with a mean age of 79 years (range 57-93 years) at death. Only specimens without evidence of previous surgery were included and all measurements were taken using electronic Tresna point digital callipers (Series SC02, Guilin, China; accuracy $\pm 0.03 \mathrm{~mm}$ and resolution $0.01 \mathrm{~mm}$ ).

\section{Dissection}

Skin and subcutaneous tissue were excised from the thigh to expose the inguinal ligament, femoral triangle and associated structures (AL, pectineus, the femoral artery, vein and nerve, sartorius and rectus femoris). Sartorius was separated and reflected superiorly to expose $\mathrm{AL}, \mathrm{AB}$ and gracilis. The epimysium investing each muscle was removed, taking care not to damage any connections between tendons, and then each muscle was detached from its pubic enthesis using sharp dissection. The shape of each tendinous footprint was recorded along with its maximum height and width.

The dimensions of the tendons and MTJs of each muscle were documented. Tendon length was measured from the enthesis to the most distal extent of the MTJ (surface or intramuscular). The MTJ was defined as the region over which muscle fibres merged with the tendon. ${ }^{40}$ The extent of the MTJ was determined by examining the superficial and deep surfaces of the muscle, reflecting if necessary any superficial fascicles to expose an intramuscular tendon. In a small sample $(n=5)$ it was possible to measure the femur length, from the anterior superior iliac spine to the medial side of the medial knee joint line; ${ }^{41}$ this measurement was used to standardise intramuscular tendon length by expressing it as a percentage of femur length.

Each musculotendinous complex was then divided into segments using a standardised approach: each tendon was divided $3 \mathrm{~mm}$ distal to the enthesis and at the midpoint of the MTJ; this segment was then bisected transversely, producing a proximal and distal tissue block for histological processing (figure 1).

\section{Histology}

Each tissue block ( $n=180$ from 20 limbs) and its associated enthesis ( $\mathrm{n}=60$ from 20 limbs) were postfixed in $10 \%$ neutral-buffered formalin and processed through to paraffin. Four $4-\mu \mathrm{m}$ thick sections were obtained. The first was taken from the enthesis itself and stained with H\&E and Alcian blue to identify fibrocartilage; $;^{42}$ the fibrocartilage

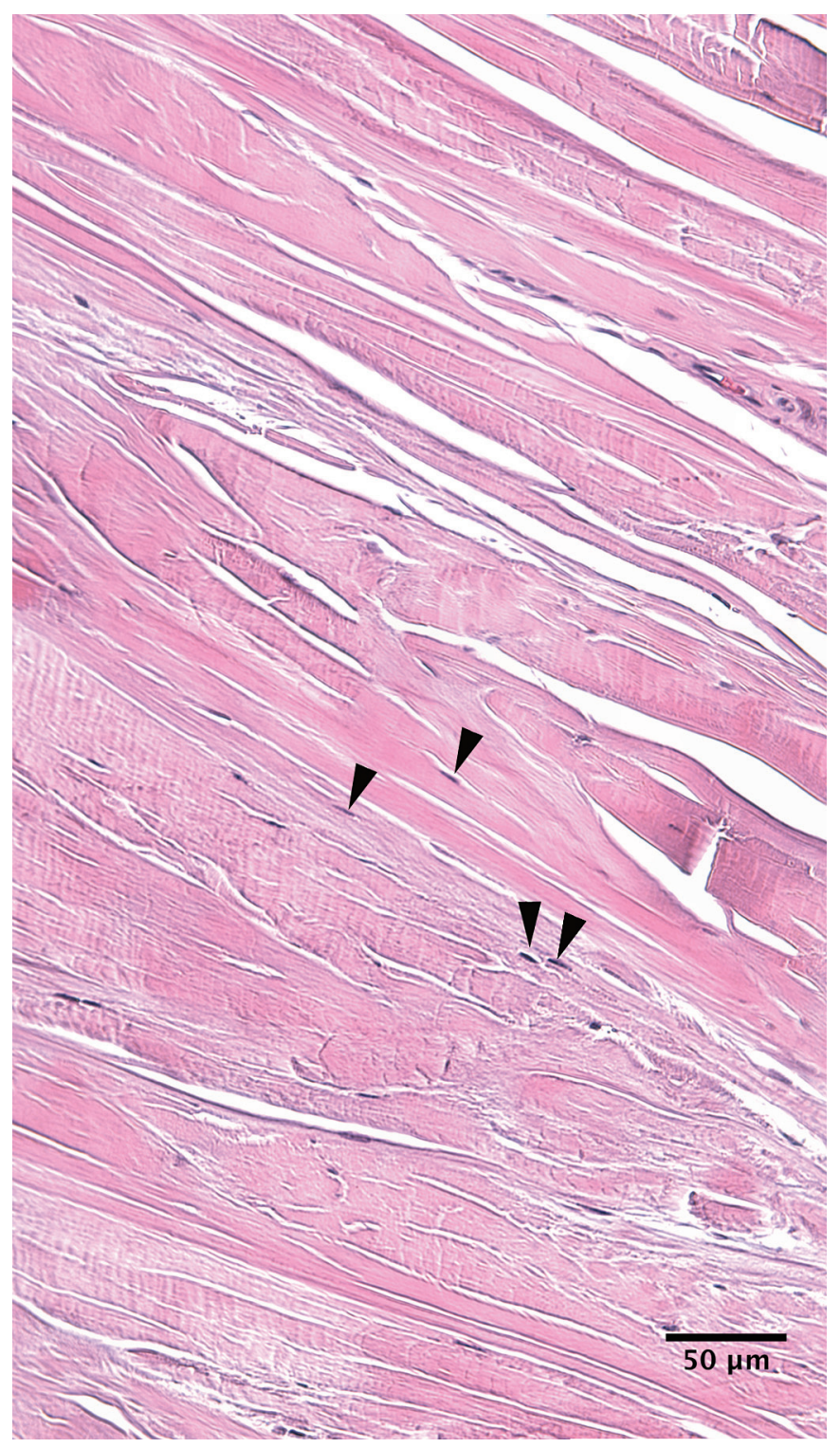

Figure 3 Photomicrograph showing fibrocartilage at the enthesis of adductor brevis (H\&E with Alcian blue stain). Chondrocytes are indicated by arrowheads.

appears as rows of chondrocytes embedded within parallel bundles of collagen fibres. ${ }^{38}$ The remaining three sections (slices $\mathrm{A}-\mathrm{C}$ ) were taken at $3 \mathrm{~mm}$ distal to the enthesis (A), mid-MTJ (C) and halfway between these two points (mid-tendon, B) (figure 1) and stained with Verhoeff-van 
Table 2 Adductor tendon and blood vessel CSA

\begin{tabular}{|c|c|c|c|c|c|}
\hline \multirow[b]{2}{*}{ Muscles } & \multirow[b]{2}{*}{ Slices } & \multirow{2}{*}{$\begin{array}{l}\text { Total section CSA }\left(\mathrm{mm}^{2}\right) \\
\text { Raw }\end{array}$} & \multicolumn{2}{|c|}{ Tendon CSA (mm²) } & \multirow{2}{*}{$\begin{array}{l}\text { Blood vessel CSA }\left(\mathrm{mm}^{2}\right) \\
\% \text { of tendon CSA }\end{array}$} \\
\hline & & & Raw & $\%$ of total CSA & \\
\hline & Slice A & $56.6 \pm 23.1$ & $50.8 \pm 18.5$ & $93.9 \pm 18.0$ & $0.3 \pm 0.3$ \\
\hline & Slice B & $220.2 \pm 120.9$ & $20.8 \pm 5.9^{* *}$ & $12.5 \pm 7.7^{* *}$ & $0.7 \pm 0.6^{*}$ \\
\hline Adductor lo & Slice C & $383.0 \pm 234.1$ & $11.5 \pm 7.3^{* *}$ & $3.4 \pm 2.3^{* *}$ & $1.1 \pm 0.6^{* *}$ \\
\hline & Slice A & $65.0 \pm 32.1$ & $48.7 \pm 19.7$ & $82.8 \pm 23.7$ & $0.5 \pm 0.4$ \\
\hline & Slice B & $226.7 \pm 82.1$ & $27.8 \pm 10.6^{* *}$ & $13.1 \pm 5.1^{* *}$ & $1.1 \pm 0.8^{*}$ \\
\hline Adductor brevis & Slice C & $319.4 \pm 134.8$ & $16.1 \pm 11.3^{* *}$ & $5.5 \pm 3.5^{* *}$ & $1.6 \pm 1.1^{*}$ \\
\hline & Slice A & $71.3 \pm 28.1$ & $61.1 \pm 22.0$ & $88.4 \pm 14.7$ & $0.9 \pm 0.6$ \\
\hline & Slice B & $72.4 \pm 25.7$ & $41.9 \pm 13.5^{* *}$ & $65.9 \pm 28.8^{* *}$ & $1.3 \pm 0.7^{*}$ \\
\hline Gracilis & Slice C & $154.3 \pm 65.7$ & $18.9 \pm 15.3^{* *}$ & $18.7 \pm 24.6^{* *}$ & $0.9 \pm 0.5$ \\
\hline
\end{tabular}

${ }^{*}$ Statistically significant values $(\mathrm{p}<0.05)$.

${ }^{*}$ *Statistically significant values $(p<0.005)$ within muscles. Slice A was taken $3 \mathrm{~mm}$ distal to the enthesis, Slice B at midtendon and slice $\mathrm{C}$ at mid-MTJ.

CSA, cross-sectional area.

Gieson. ${ }^{38} 42$ Stained sections were mounted in DPX, coverslipped and examined under an Olympus AX70 light microscope (Olympus, Tokyo, Japan).

QCapture software (QCapture 3.0; OImaging, Surrey, Canada) and a digital camera (MicroPublisher 5.0 RTV; OImaging) were used to take photomicrographs, which were then assembled into a montage using Photoshop CS3 Extended Version 10.0 (Adobe Systems Incorporated, San Jose, California, USA), and analysed using ImageJ (National Institutes of Health, Bethesda, Maryland, USA). The 'polygon section tool' was used to draw a line around the perimeter of the entire section and, within sections of the MTJ, around the tendinous portion; in this way, both the total CSA of the slice and the proportion occupied by tendon could be calculated. Within the tendinous area, all blood vessels with a maximum diameter of $20 \mu \mathrm{m}$ or more were identified and their total combined CSA was calculated. ${ }^{43}$ Blood vessel CSA was then expressed as a percentage of tendon CSA.

\section{Statistical analysis}

Measurement reliability of CSA and vessel count was assessed by reanalysing 10 randomly selected sections. Intraclass correlation coefficients were calculated (SPSS version 18; SPSS Inc, Chicago, Illinois, USA) and interpreted using the criteria of Landis and Koch (1977). ${ }^{44}$

To compare vascularity between the three tendon levels, data for mean total CSA, tendon CSA and blood vessel CSA from each slice (A-C) were entered into Microsoft Excel (2008) and compared using paired (within samples) or unpaired (between samples) $t$ tests. $p$ values less than 0.05 are considered statistically significant.

\section{RESULTS}

\section{Dissection}

The proximal attachments of $\mathrm{AL}$ and $\mathrm{AB}$ extended from the anterior surface of the pubic body inferior to the pubic tubercle. For AL this was in a superior direction partially covering the insertion of $A B$ while the attachment of $A B$ extended down the inferior pubic ramus where it fused with the proximal tendon of gracilis. The shapes and dimensions of each entheseal footprint varied (table 1).
$A B$ and gracilis were fused proximally in all specimens. In males, $A B$ muscle fibres inserted into the deep aspect of the gracilis tendon (figure $2 \mathrm{~A}$ ), whereas in females, the tendons fused to form a common tendon that inserted into the inferior pubic ramus (figure $2 \mathrm{~B}$ ). The proximal tendons of AL and gracilis were fused in one male specimen (figure 2C); in all others they were separate. The pattern of fusion was symmetrical in all individuals.

Some of the proximal anterior fibres of the large AL tendon inserted into the capsular tissues of the pubic symphysis and were continuous with rectus abdominis (figure 2D). Little substantial tendon was evident on the surface of $A B$ and many of its muscle fibres inserted directly into bone. The proximal tendon of gracilis was short, wide and thin (table 1); in six specimens it decussated and fused with the capsular tissues of the pubic symphysis (figure 2D).

An extensive intramuscular tendon was present in all $\mathrm{AL}$ and $A B$ muscles. With the exception of $A L$ in one specimen, these intramuscular tendons extended further distally than the superficial tendon (table 1). The intramuscular tendons of $\mathrm{AL}$ and $\mathrm{AB}$ measured $23 \% \pm 3 \%$ and $11 \% \pm 2 \%$ of femur length, respectively. The length of the MTJs corresponded to tendon length such that the MTJ of AL was the longest, while that of $A B$ and gracilis were substantially shorter (table 1). Gracilis had no intramuscular tendon.

\section{Histology}

All entheses were fibrocartilaginous (figure 3). A representative series of histological sections through the proximal portion of $\mathrm{AB}$ (slices $\mathrm{A}-\mathrm{C}$ ) is shown in figure 4. Data on tendon CSA and relative vascularity are shown in table 2 . Intraclass correlation coefficients ranged between 0.63 and 1.00 , indicating substantial to perfect agreement between repeated measures. ${ }^{44}$

The CSA of the proximal tendons of $\mathrm{AL}, \mathrm{AB}$ and gracilis decreased significantly from slices $A$ to $C(p \leq 0.001)$. The only significant gender difference was in AL tendon morphology: in male specimens, $80 \%$ of slice $\mathrm{A}$ ( $3 \mathrm{~mm}$ from the enthesis) comprised tendon with muscle constituting most of the remaining CSA at this point, whereas in female specimens $99.9 \%$ of slice A was tendon $(p<0.05)$. The vascularity of the proximal tendons of $A L$ and $A B$ decreased significantly at each level nearer 

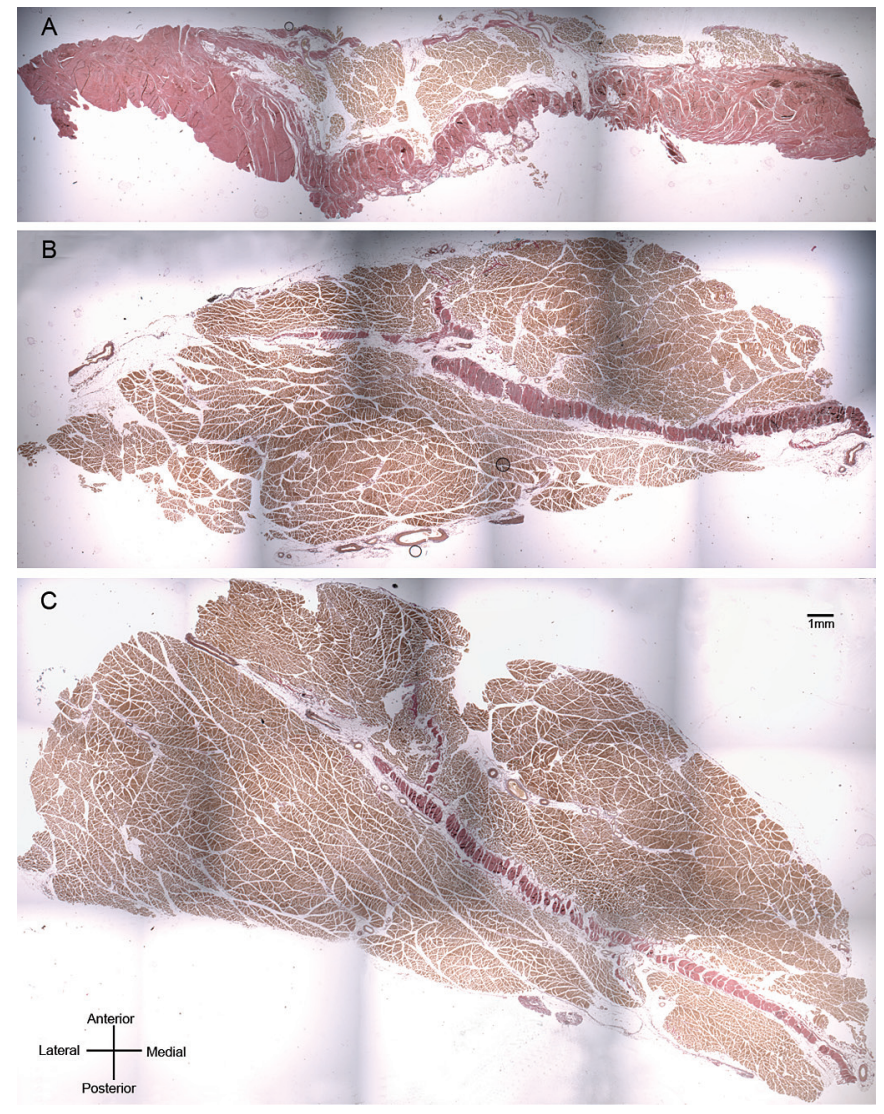

Figure 4 Photomicrographs showing the cross-sectional anatomy of a left AB muscle (Verhoeff-van Gieson stain). Sections taken 3 mm from the enthesis (A), at mid-tendon (B) and at mid-MTJ (C). There is a prominent intramuscular tendon (pink) in each slice. Muscle is stained brown. $A$ similar intramuscular tendon was present in $A L$ but not gracilis. $A B$, adductor brevis; $A L$, adductor longus; MTJ, musculotendinous junction.

the enthesis (AL $p<0.001 ; A B p<0.05)$. In contrast, the vascularity of the proximal tendon of gracilis was greater in the mid-tendon compared to proximal and distal levels (proximal $\mathrm{p}=0.0017 ;$ distal $\mathrm{p}=0.057$ ).

Near the enthesis (slice A), AL and AB were both significantly relatively less vascular than gracilis (AL $p<0.001 ; A B$ $p<0.05)$. There was no significant difference between the relative vascularity of $A L$ and $A B$ at this site. At the mid-tendon (slice B), AL was relatively less vascular than gracilis $(p<0.005)$ and at the mid-MTJ (slice C), AB was significantly more vascular than gracilis $(\mathrm{p}<0.05)$ (figure 5$)$.

\section{DISCUSSION}

This study reveals several novel features about the proximal segments of $\mathrm{AL}, \mathrm{AB}$ and gracilis, which may be relevant to the pathogenesis of groin strain. In all specimens, entheses were found to be fibrocartilaginous, either the muscle (in males) or the tendon (in females) of $\mathrm{AB}$ was fused to the proximal tendon of gracilis, and an intramuscular tendon was identified within both $\mathrm{AL}$ and $\mathrm{AB}$. In addition, we found statistically significant differences in the vascularity of each proximal tendon; the vascularity of the proximal tendons of $A L$ and $A B$ decreased significantly nearer the enthesis and both were relatively less vascular than gracilis at this site.

While the enthesis of $\mathrm{AL}$ is understood to be fibrocartilaginous, ${ }^{14}$ the nature of the entheses of $A B$ and gracilis has not been previously examined. Nevertheless, our findings are not unexpected since fibrocartilaginous entheses are more common than fibrous types, their rows of collagen bundles oriented in the direction of stress to provide additional

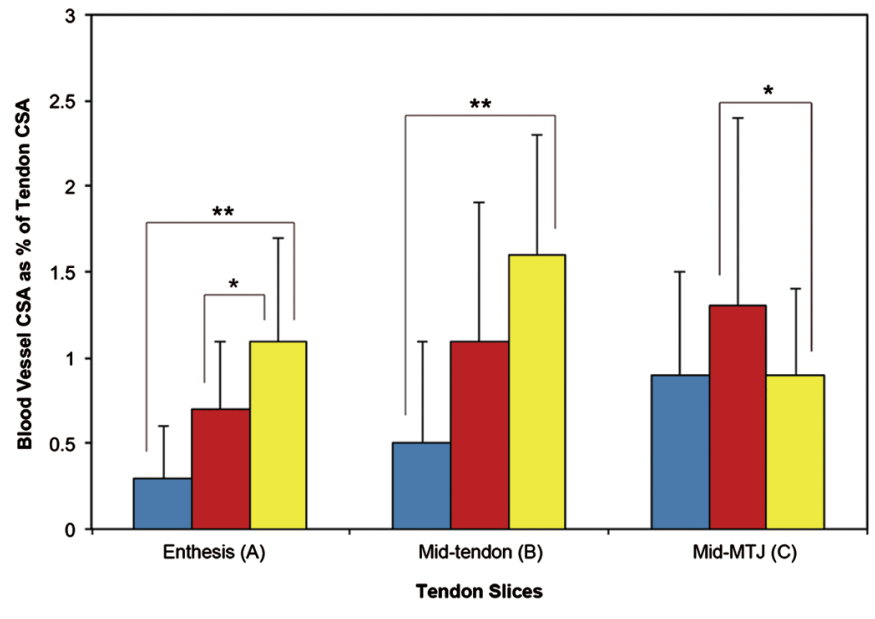

Figure 5 Relative differences in vascularity between the proximal tendons of $A L, A B$ and gracilis. The coloured bars represent mean data from $A L$ (blue), $A B$ (red) and gracilis (yellow). Sections taken 3 $\mathrm{mm}$ from the enthesis $(A)$, at the mid-tendon $(B)$ and at the mid-MTJ (C). ${ }^{*}$ Statistically significant difference $(p<0.05),{ }^{* *}(p<0.005)$. $A B$, adductor brevis; $A L$, adductor longus; MTJ, musculotendinous junction.

strength. ${ }^{4546}$ Further, the amount of fibrocartilage at an enthesis directly correlates with the degree of compressive stress, the fibrocartilage helping to protect the tendon from compression and the bone from excessive shear. Fibrocartilage may also protect the tendon vasculature. ${ }^{46}$ 
A distinctive intramuscular tendon was present in both AL and AB. A deep tendinous extension within the muscle belly forming an elongated MTJ has been demonstrated in other limb muscles, including the hamstrings, 404748 but we could not find any previous descriptions of intramuscular tendons within the adductors. Functionally, an intramuscular tendon may provide extra strength and stability during muscle contraction and could be especially important in muscles such as AL whereby the MTJ is subjected to large mechanical stresses. ${ }^{31} 32$ From a clinical perspective, adductor injury can occur at the proximal tendon or MTJ, 111215 16; awareness of the existence of an intramuscular tendon could therefore be important in diagnostic imaging. It is likely that previous reports of tendon and MTJ injuries affecting $\mathrm{AL}$ and $\mathrm{AB}$ have been limited to their surface tendons, ${ }^{15} 1627$ and pathology affecting the intramuscular tendinous component may have been overlooked.

To our knowledge, this is the first study to investigate the relative vascularity of the proximal adductor tendons. AL and $A B$ were less vascular nearer the enthesis, a factor which may adversely influence the capacity or rate of tendon repair in $\mathrm{AL}$ and $\mathrm{AB} \cdot{ }^{45}$ In contrast to $\mathrm{AL}$ and $\mathrm{AB}$, the mid-tendon of gracilis was found to be significantly more vascular than its enthesis or mid-MTJ region, and gracilis was relatively more vascular than $A L$ and $A B$ at its enthesis. It has previously been hypothesised that poor entheseal blood supply could contribute to adductor-related groin pain, ${ }^{21}$ but, conversely, it has been shown that vascularity increases with enthesopathy. ${ }^{45}$ If vascularity is an important factor in proximal adductor tendon injury and repair, this could have implications for adductorrelated groin strain. However, further research is needed to verify our observations, ascertain the topographical patterns of injury within the proximal segments of the adductor muscles, and determine if vascularity is a significant factor in the pathogenesis and/or prognosis of this condition.

The proximal anatomy of gracilis has been studied before as the muscle is often used for reconstructive surgery, ${ }^{49} 50$ but its relationship to adjacent muscles remains controversial. Fusion between the proximal tendons of gracilis and AL was evident in only one of our ten cadavers. This finding is consistent with most descriptions in anatomical texts, ${ }^{35-37} 51$ but is at odds with two MRI studies which stated that gracilis and AL fused to form a converging aponeurosis. ${ }^{2} 33$ These disparities may reflect individual anatomical variation or differences in study methods. An interesting finding in our study, documented only once before in the literature, ${ }^{29}$ was the discovery of fusion between proximal gracilis and $A B$ in all specimens. In a study of seven cadavers, Budinoff and Tague (1990) found that AB and gracilis shared a common insertion site, ${ }^{29}$ although they made no comment on possible gender differences in this union. While our findings must be tentative because of the imbalance between male and female specimens in our study, we observed that $\mathrm{AB}$ muscle fibres inserted into the deep aspect of the gracilis tendon in males, but there was tendinous fusion in females. These differences are consistent with our histological findings that showed up to $20 \%$ of the CSA of the tendon was occupied by muscle that was $3 \mathrm{~mm}$ from the enthesis in males, whereas it was totally tendinous at this site in females. The relevance of these findings to adductor-related groin strain is uncertain. It is known that these injuries are more common in males $^{752}$ but whether this is because of differences in sporting activities or biological differences is unclear.

Our study has several limitations. Firstly, we only had access to elderly embalmed cadavers, whose activity in life was unknown. It would have been preferable to have included a larger proportion of male specimens, since adductor-related groin pain is more common in male athletes ${ }^{7} 52$ Moderately large variations in muscle bulk were evident between specimens, and this was reflected by the SDs of the data. In addition, some muscle shrinkage occurs during or after embalming, but whether tendon shrinks to the same degree is unknown. ${ }^{53}$ Further research including larger numbers of younger cadavers would be useful to confirm our findings. While our method of assessing tendon vascularity was similar to a previous study, ${ }^{43}$ it only assessed the relative proportion of the CSA of the tendon occupied by blood vessels above a threshold size. We did not attempt to separately quantify arteries and veins, and we do not know whether the total blood vessel CSA we calculated necessarily reflects blood flow in vivo. Finally, our novel observations require validation using imaging techniques in both healthy individuals and patients with groin strain. It would be particularly interesting to investigate the latter to determine if the intramuscular tendons in AL and AB show evidence of pathology and whether, using colour Doppler ultrasound imaging, there are demonstrable changes in blood flow to the tendon in the region of the proximal enthesis.

\section{CONCLUSIONS}

This study describes the anatomy of the proximal portions of the adductor muscles, $\mathrm{AL}, \mathrm{AB}$, and gracilis. We have shown that all three muscles have a fibrocartilaginous enthesis and that intramuscular tendons are a constant feature of $\mathrm{AL}$ and $A B$, but not gracilis. In addition, the proximal tendons of $A B$ and gracilis are fused. The proximal tendons of $A L$ and $A B$ are less vascular towards the enthesis. These anatomical findings need to be considered as possible factors contributing to the pathogenesis and pattern of injuries in adductor-related groin strain.

Acknowledgements The authors thank Amanda Fisher, Histology Services Unit, Otago School of Medical Sciences, for her expert assistance with histology and Andrew McNaughton, Otago Centre for Confocal Microscopy, for his guidance with microscopy and ImageJ.

Competing interests None.

Provenance and peer review Not commissioned; externally peer reviewed

\section{REFERENCES}

1. Arnason A, Sigurdsson SB, Gudmundsson A, et al. Risk factors for injuries in football. Am J Sports Med 2004;32:5-16S.

2. Cunningham PM, Brennan D, O'Connell M, et al. Patterns of bone and soft-tissue injury at the symphysis pubis in soccer players: observations at MRI AJR Am J Roentgenol 2007;188:W291-6.

3. Engström B, Forssblad M, Johansson C, et al. Does a major knee injury definitely sideline an elite soccer player? Am J Sports Med 1990;18:101-5.

4. Ekstrand J, Hägglund $\mathrm{M}$, Waldén $\mathrm{M}$. Epidemiology of muscle injuries in professional football (soccer). Am J Sports Med 2011;39:1226-32.

5. Emery CA, Meeuwisse WH. Risk factors for groin injuries in hockey. Med Sci Sports Exerc 2001;33:1423-33.

6. Orchard J, Seward H. Epidemiology of injuries in the Australian Football League, seasons 1997-2000. Br J Sports Med 2002;36:39-44.

7. Paajanen $\mathbf{H}$, Ristolainen $\mathrm{L}$, Turunen $\mathrm{H}$, et al. Prevalence and etiological factors of sport-related groin injuries in top-level soccer compared to non-contact sports. Arch Orthop Trauma Surg 2011;131:261-6.

8. Robertson BA, Barker PJ, Fahrer M, et al. The anatomy of the pubic region revisited: implications for the pathogenesis and clinical management of chronic groin pain in athletes. Sports Med 2009;39:225-34.

9. Hölmich P. Long-standing groin pain in sportspeople falls into three primary patterns, a "clinical entity" approach: a prospective study of 207 patients. Br J Sports Med 2007;41:247-52; discussion 252

10. Werner $\mathbf{J}$, Hägglund $\mathrm{M}$, Waldén $\mathrm{M}$, et al. UEFA injury study: a prospective study of hip and groin injuries in professional football over seven consecutive seasons. Br J Sports Med 2009:43:1036-40. 
11. Gabbe BJ, Bailey M, Cook JL, et al. The association between hip and groin injuries in the elite junior football years and injuries sustained during elite senior competition. Br J Sports Med 2010;44:799-802.

12. Zoga AC, Kavanagh EC, Omar IM, et al. Athletic pubalgia and the "sports hernia": MR imaging findings. Radiology 2008;247:797-807.

13. Dimitrakopoulou A, Schilders EM, Talbot JC, et al. Acute avulsion of the fibrocartilage origin of the adductor longus in professional soccer players: a report of two cases. Clin J Sport Med 2008;18:167-9.

14. Ippolito E, Postacchini F. Rupture and disinsertion of the proximal attachment of the adductor longus tendon. Case report with histochemical and ultrastructura study. Ital J Orthop Traumatol 1981;7:79-85

15. Atkinson HD, Johal P, Falworth MS, et al. Adductor tenotomy: its role in the management of sports-related chronic groin pain. Arch Orthop Trauma Surg 2010:130:965-70.

16. Kälebo $\mathbf{P}$, Karlsson J, Swärd L, et al. Ultrasonography of chronic tendon injuries in the groin. Am J Sports Med 1992;20:634-9.

17. Martens MA, Hansen L, Mulier JC. Adductor tendinitis and musculus rectus abdominis tendopathy. Am J Sports Med 1987;15:353-6.

18. Renström P, Peterson L. Groin injuries in athletes. Br J Sports Med 1980;14:30-6

19. Robertson IJ, Curran C, McCaffrey N, et al. Adductor tenotomy in the management of groin pain in athletes. Int J Sports Med 2011;32:45-8.

20. Schilders $\mathbf{E}$, Bismil 0 , Robinson $\mathrm{P}$, et al. Adductor-related groin pain in competitive athletes. Role of adductor enthesis, magnetic resonance imaging, and entheseal pubic cleft injections. J Bone Joint Surg Am 2007:89:2173-8.

21. Holmich P. Adductor-related groin pain in athletes. Sports Med Arthrosc Rev 1997:5:285-91.

22. Akermark C, Johansson C. Tenotomy of the adductor longus tendon in the treatment of chronic groin pain in athletes. Am J Sports Med 1992;20:640-3.

23. Ekstrand J, Hilding J. The incidence and differential diagnosis of acute groin injuries in male soccer players. Scand J Med Sci Sports 1999;9:98-103.

24. Hölmich P, Hölmich LR, Bjerg AM. Clinical examination of athletes with groin pain: an intraobserver and interobserver reliability study. Br J Sports Med 2004;38:446-51.

25. Schilders E, Talbot JC, Robinson P, et al. Adductor-related groin pain in recreational athletes: role of the adductor enthesis, magnetic resonance imaging and entheseal pubic cleft injections. J Bone Joint Surg Am 2009;91:2455-60.

26. Robinson $\mathbf{P}$, Barron DA, Parsons W, et al. Adductor-related groin pain in athletes: correlation of MR imaging with clinical findings. Skeletal Radiol 2004;33:451-7.

27. Attarian DE. Isolated acute hip adductor brevis strain. J South Orthop Assoc 2000:9:213-15.

28. Pedret $\mathbf{C}$, Balius $\mathrm{R}$, Barceló $\mathrm{P}$, et al. Isolated tears of the gracilis muscle. Am J Sports Med 2011;39:1077-80.

29. Budinoff LC, Tague RG. Anatomical and developmental bases for the ventral arc of the human pubis. Am J Phys Anthropol 1990:82:73-9.

30. Robinson P, Salehi F, Grainger A, et al. Cadaveric and MRI study of the musculotendinous contributions to the capsule of the symphysis pubis. AJR Am J Roentgenol 2007;188:W440-5.

31. Strauss EJ, Campbell K, Bosco JA. Analysis of the cross-sectional area of the adductor longus tendon: a descriptive anatomic study. Am J Sports Med 2007;35:996-9
32. Tuite DJ, Finegan PJ, Saliaris AP, et al. Anatomy of the proximal musculotendinous junction of the adductor longus muscle. Knee Surg Sports Traumatol Arthrosc 1998;6:134-7.

33. Brennan D, O'Connell MJ, Ryan M, et al. Secondary cleft sign as a marker of injury in athletes with groin pain: MR image appearance and interpretation. Radiology 2005;235:162-7.

34. Koulouris $\mathbf{G}$. Imaging review of groin pain in elite athletes: an anatomic approach to imaging findings. AJR Am J Roentgenol 2008;191:962-72.

35. Standring S, ed. Gray's Anatomy: The Anatomical Basis of Clinical Practice. 40th edition. Edinburgh: Churchill Livingstone 2008.

36. Moore KL, Dalley AF, Agur AMR. Clinically Oriented Anatomy. Sixth edition. Philadelphia, PA: Lippincott Williams \& Williams 2010:448-51.

37. Rosse C, Gaddum-Rosse P. Hollinshead's Textbook of Anatomy. Fifth edition. Philadelphia, PA: Lippincott-Raven Publishers 1997:363-8.

38. Stevens A, Lowe JS. Human Histology. Third edition. St Louis, MO: Elsevier Mosby 2005:249.

39. Omar IM, Zoga AC, Kavanagh EC, et al. Athletic pubalgia and "sports hernia": optimal MR imaging technique and findings. Radiographics 2008;28:1415-38.

40. Woodley SJ, Mercer SR. Hamstring muscles: architecture and innervation. Cells Tissues Organs (Print) 2005;179:125-41.

41. Thomeé R, Renström P, Karlsson J, et al. Patellofemoral pain syndrome in young women. I. A clinical analysis of alignment, pain parameters, common symptoms and functional activity level. Scand J Med Sci Sports 1995;5:237-44.

42. Young B, Lowe JS, Stevens A, et al. Wheater's Functional Histology: A Text and Colour Atlas. Fifth edition. Edinburgh: Churchill Livingstone/Elsevier 2006:189.

43. Carr AJ, Norris SH. The blood supply of the calcaneal tendon. J Bone Joint Surg Br 1989;71:100-1.

44. Landis JR, Koch GG. The measurement of observer agreement for categorical data. Biometrics 1977:33:159-74.

45. Benjamin M, Toumi H, Ralphs JR, et al. Where tendons and ligaments meet bone: attachment sites ('entheses') in relation to exercise and/or mechanical load. J Anat 2006;208:471-90

46. Benjamin M, Ralphs JR. Fibrocartilage in tendons and ligaments-an adaptation to compressive load. J Anat 1998;193 (Pt 4):481-94

47. Segal RL, Catlin PA, Krauss EW, et al. Anatomical partitioning of three human forearm muscles. Cells Tissues Organs (Print) 2002;170:183-97.

48. Segal RL, Wolf SL, DeCamp MJ, et al. Anatomical partitioning of three multiarticular human muscles. Acta Anat (Basel) 1991:142:261-6.

49. Coquerel-Beghin D, Milliez PY, Auquit-Auckbur l, et al. The gracilis musculocutaneous flap: vascular supply of the muscle and skin components. Surg Radiol Anat 2006;28:588-95.

50. Macchi V, Vigato E, Porzionato A, et al. The gracilis muscle and its use in clinical reconstruction: an anatomical, embryological, and radiological study. Clin Anat 2008:21:696-704.

51. Palastanga N, Field D, Soames R. Anatomy and Human Movement: Structure and Function. Third edition. Oxford: Butterworth-Heinemann 1998:337.

52. Hägglund $\mathbf{M}$, Waldén $\mathbf{M}$, Ekstrand J. Injuries among male and female elite football players. Scand J Med Sci Sports 2009;19:819-27.

53. Cutts A. Shrinkage of muscle fibres during the fixation of cadaveric tissue. J Anat 1988;160:75-8. 